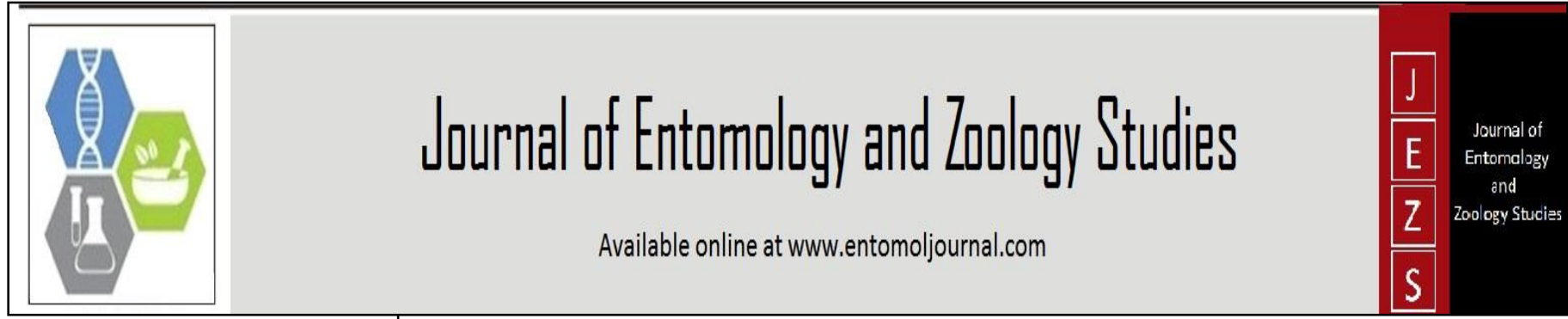

E-ISSN: 2320-7078

P-ISSN: 2349-6800

www.entomoljournal.com

JEZS 2021; 9(3): 346-347

(C) 2021 JEZS

Received: 22-03-2021

Accepted: 24-04-2021

\section{K Jeyaraja}

Professor, Department of

Veterinary Clinical Medicine

Madras Veterinary College,

Chennai, Tamil Nadu, India

\section{KS Saikrishna}

Ph.D Scholar, Department of

Veterinary Clinical Medicine,

Madras Veterinary College,

Chennai, Tamil Nadu, India

\section{E Venkatesa Kumar}

Assistant Professor and Head,

Department of Veterinary

Clinical Medicine, Veterinary

College and Research Institute,

Namakkal, Tamil Nadu, India

\section{R Ramprabhu}

Professor and Head, Department of Veterinary Clinical Complex, Veterinary College and Research Institute, Tirunelveli, Tamil Nadu, India

\section{Corresponding Author:}

Ph.D Scholar, Department of Veterinary Clinical Medicine,

Madras Veterinary College,

Chennai, Tamil Nadu, India

\section{Botulism in dogs: A clinical case study}

\section{K Jeyaraja, KS Saikrishna, E Venkatesa Kumar and R Ramprabhu}

DOI: https://doi.org/10.22271/j.ento.2021.v9.i3e.8726

\section{Abstract}

Botulism is a rare and fatal disease of humans and animals caused by the bacterium Clostridium botulinum that produces the botulinum toxin responsible for flaccid paralysis. The present case study discusses about the clinical signs, diagnostic evaluation and therapeutic outcome of carrion associated botulism in dogs. A total of eight dogs were presented with signs of dysphagia, regurgitation, incoordination in gait and recumbency in some cases after feeding with poultry abattoir waste. Radiographic findings revealed megaesophagus with ingested bone pieces in stomach of five dogs. Based on the clinical signs, anamnestic data, and radiographic findings the cases were tentatively diagnosed as carrion botulism and was successfully managed.

Keywords: carrion botulism, dogs, lower motor neuron disease, megaesophagus

\section{Introduction}

Canine botulism was first reported in 1978 in the United States of America. It is a rare and fatal neurotoxic disease of humans, animals, and birds that causes flaccid muscular paralysis. Botulinum is the exotoxin produced by the gram-positive anaerobic bacteria Clostridium botulinum which has seven different serotypes ranging from A-G. Of these A, B, E and F are responsible for human botulism ${ }^{[4]}$ whereas type $\mathrm{C}$ has been majorly implicated for botulism in dogs. Most commonly exposure of animals to these toxins occurs by ingestion of preformed toxins in food (Carrion ingestion). In addition, wound botulism and intestinal botulism can also occur in which organism multiply and secrete toxins in wounds and intestines, respectively ${ }^{[5]}$. In a study ${ }^{[7]}$ about $40 \%$ of the neuromuscular disorders in dogs were recorded to be affected by botulism. Owing to the unavailability of toxicological studies in the field, diagnosis of botulism is mainly done by clinical correlation of history, clinical signs and diagnostic findings. Available literature was scanty regarding studies on botulism in dogs. The present case study describes the clinical signs, diagnostic approach and management of canine botulism.

\section{Materials and Methods}

Eight dogs of different breeds which includes six native Tamil Nadu breeds and two nondescript breeds belonging to different age groups were presented with the signs of dysphagia, regurgitation, muscular tremors, ataxic gait (Fig.1) and hind limb paresis. Detailed anamnestic data revealed dietary inclusion of poultry abattoir waste predominantly with leftover bony material which was identified as the common inciting factor in all the affected ones. This abattoir waste was being fed to the dogs as it is available ad libitum and cost-free to the owners. Initial clinical signs recorded were hindlimb weakness in 6 cases whereas the remaining 2 dogs had shown hind limb paralysis. Dysphagia and regurgitation were noticed within 2-4 days after the development of initial signs.

Neurological examination revealed flaccidity in hindlimb muscles with loss of superficial pain reflex. Cough was also evident in three of the affected dogs. Detailed haematological, serum biochemical and radiographic examination was performed. The diagnostic findings and therapy were detailed below.

\section{Results and Discussion}

Haematological and serum biochemical findings revealed no significant abnormality in all the affected cases. Plain radiography findings revealed megaesophagus along with the ingested 
bones (Fig.2) in five of the affected cases. Animals were treated with Inj. RL IV, Inj. Amoxicillin and Sulbactam @ $15 \mathrm{mg} / \mathrm{kg}$ IV and Injectable nerve stimulant containing Pyridoxine hydrochloride, Mecobalamin and Nicotinamide @ $2 \mathrm{ml}$ IV for 5 days. Significant clinical improvement was noticed in all the eight cases from third day with complete resolution by fifth day in six cases and by eighth day in two cases. No reoccurrence of signs was noticed after the therapy. Owners were advised to avoid feeding abattoir waste to the dogs.

Ingestion of preformed toxins is more common than toxicoinfection in animals wherein ingested preformed toxin will be absorbed from intestines and reaches the bloodstream. Earlier studies revealed that carrions were a potential source of infection to companion animals as they have frequent access to it. Botulinum was often considered the strongest microbial toxin as it even resists proteolytic degradation in the gastrointestinal tract ${ }^{[5]}$. This toxin once absorbed will block the release of acetylcholine from presynaptic vesicles and thus the impulse conduction through the synapse and muscular contraction will be hindered leading to flaccid paralysis. In the present case study, it was noted that the dogs were fed with the poultry abattoir waste which predominantly included bones with leftover meat on them that acted as a toxin source. The presence of bone material in the radiography also confirmed the ingestion of the suspected material. Poultry waste and raw meat act as a potential source for botulism in cattle and dogs, respectively ${ }^{[2,6]}$.
The differential diagnosis for botulism in dogs includes paralytic form of rabies, polyradiculoneuritis and myasthenia gravis. Though confirmatory diagnosis for botulism is through demonstration of the toxin by serum neutralisation tests in mice ${ }^{[3]}$ the non-availability of test under field conditions forces the clinician to adapt presumptive diagnosis basing on the history and clinical signs ${ }^{[1]}$. Antitoxins are reportedly effective in the early phase of disease when there are free circulatory toxins however the poor availability of antitoxin as well high therapeutic cost restricts its usage in veterinary practice.

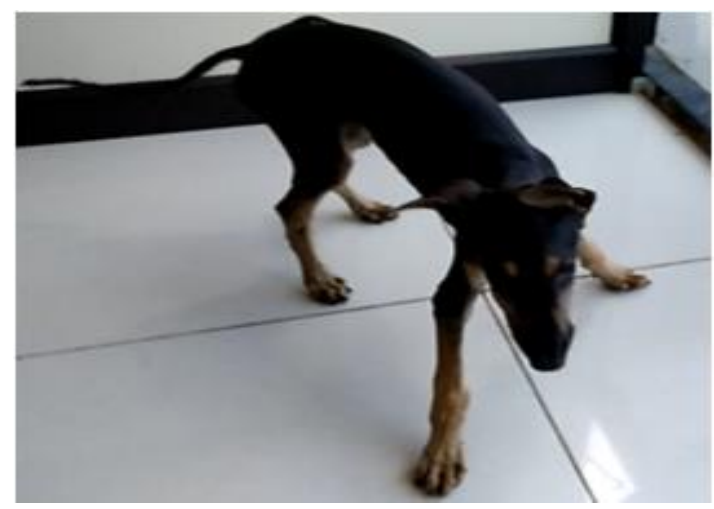

Fig 1: Dog showing ataxic gait

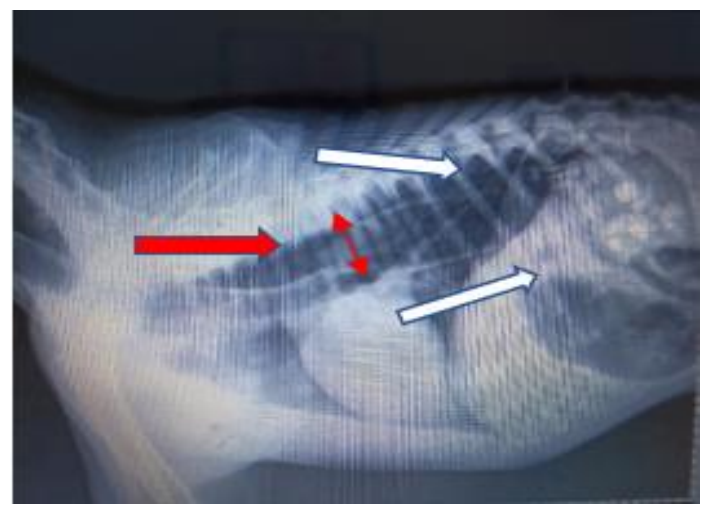

Fig 2: Plain radiograph indicating megaesophagus (red arrow) and ingested bone pieces of fowl in esophagus and stomach (white arrows)

In conclusion, owing to high probability of infecting with botulism it was recommended to avoid feeding abattoir waste/poultry carcass to dogs as it may result in potential complications with botulism which if undiagnosed will result in respiratory paralysis and death.

\section{References}

1. Anniballi F, Fiore A, Lofstrom C, Skarin H, Auricchio B, Woudstra $\mathrm{C}$ et al. Management of animal botulism outbreaks: from clinical suspicion to practical countermeasures to prevent or minimize outbreaks. Biosecurity and bioterrorism: biodefense strategy, practice, and science 2013;11(S1):S191-S199.

2. Bruchim Y, Steinman A, Markovitz M, Baneth G, Elad D, Shpigel NY. Toxicological, bacteriological and serological diagnosis of botulism in a dog. Veterinary Record 2006;158:768-769.

3. Lobato FCF, Salvarani FM, Silva ROS, Souza AMD, Lima CGRD, Pires PS et al. Botulism in ruminants being fed with poultry litter. Ciencia Rural 2008;38(4):11761178.
4. Sobel J, Tucker N, Sulka A, Mclaughlin J, Maslanka S. Food borne botulism in the United States 1990-2000. Emerging Infectious Diseases 2004;10:1606-1611

5. Oliveira Junior CAD, Silva ROS, Olinda RG, Lobato FCF. Botulism in non-ruminants in Brazil. Ciencia Rural. 2016;46(12):2158-2165

6. Smart JL, Jones TO, Clegg FG, MC Murtry MJ. Poultry waste associated type $\mathrm{C}$ botulism in cattle. Epidem. Inf. 1987;98:73-79

7. Stucchi C. Avoiding misdiagnosis in patients with acute paresis/paralysis with emphasis on botulism. Revista de Ciencias Agroveterinarias 2013;13:3-4. 